\title{
Calculation of Thermodynamic Properties of Polyelectrolytes
}

\author{
R. A. MARCUS \\ Department of Chemistry, Polytechnic Institute of Brooklyn, Brooklyn 1, New York
}

(Received October 7, 1954)

\begin{abstract}
Expressions are derived which introduce an appreciable simplification into the calculation of the thermodynamic properties of solutions of polyelectrolytes in certain cases. For example, for a certain class of theoretical models of these systems it is found that the square of the mean ion activity coefficient of a uniunivalent salt in the presence of polymeric ions is $V^{2} / \int e^{-e \psi / k T} d V \cdot \int e^{e \psi / k T} d V$, the integration of the potential $\psi$ being over a region whose volume is the volume of solution per macro-ion, $V$, and whose symmetry is that assumed for the polyelectrolyte. The osmotic pressure of a salt-polyelectrolyte system is, ignoring the contribution of the macro-ion, estimated to be $\Sigma_{i} c_{i}{ }^{s} k T$, where $\Sigma_{i} c_{i}^{s}$ is the sum of the concentrations of all ions at the surface bounding the previously defined volume $V$. Other relations and various applications are given. 'The activity coefficient of salt in the presence of polyelectrolytes, calculated by extending the "parallel rod" picture of polymeric ions, is found to be in reasonable agreement with the experimental data. The use of the Poisson-Boltzmann equation to estimate $\psi$ in these systems is shown not to render inconsistent several alternative expressions for the electrostatic contribution to the free energy.
\end{abstract}

\section{INTRODUCTION}

$T$ HE strong electrostatic fields in the neighborhood of polymeric ions have been established experimentally and their theoretical description ${ }^{1-8}$ has been the subject of a number of recent communications. Relevant thermodynamic data include osmotic pressures, activity coefficients of salts, and titration behavior of polymeric acids and bases. Usually these properties are calculated theoretically by differentiation of a free-energy expression into which parameters of the models have been introduced. Because of the frequent complex dependence of these parameters on the thermodynamic variables such as the moles of the components and the volume of the solution, the differentiation may become a lengthy process. A somewhat different procedure is employed here for a certain class of models, in that the last two steps are reversed-expressions are set up for various thermodynamic properties by differentiation of the free energy, these are then simplified and the parameters are introduced as a final step. This procerure effects a considerable simplification of the calculation in various cases.

\section{THEORETICAL}

\section{General}

A number of theoretical models of polyelectrolytes have been advanced ${ }^{1-8}$; several of these have in common the following assumptions:

(1) The interaction between polymeric ions is neglected except insofar as the concentration of these ions

\footnotetext{
${ }^{1}$ Kuhn, Künzle, and Katchalsky, Helv. Chim. Acta 31, 1994 (1948).

${ }^{2}$ J. J. Hermans and J. T. G. Overbeek, Rec. trav. chim. 67, 761 (1948).

${ }^{3}$ Alfrey, Berg, and Morawetz, J. Polymer Sci. 7, 543 (1951).

${ }^{4}$ Fuoss, Katchalsky, and Lifson, Proc. Natl. Acad. Sci. U. S. 37, 579 (1951).

${ }^{5}$ Kimball, Cutler, and Samelson, J. Phys. Chem. 56, 57 (1952).

${ }^{6}$ P. J. Flory, J. Chem. Phys. 21, 162 (1953).

7 Osawa, Imai, and Kagawa, J. Polymer Sci. 13, 93 (1954).

${ }^{8}$ F. E. Harris and S. A. Rice, J. Phys. Chem. 58, 725 (1954); R. A. Marcus, J. Pliys. Cbem. 58, 621 (1954).
}

determines the size of the electrically neutral volume $V$ assigned to each polymeric ion (total volume of solution divided by number of such ions).

(2) The electrostatic interaction between all ions in this subvolume obeys the Poisson-Boltzmann equation

$$
\nabla^{2} \psi=-\frac{4 \pi}{D}\left(\rho+\rho_{p}\right)
$$

where the charge density of mobile ions $\rho$ equals $\sum_{i} c_{i} e_{i}$. The charge of ions of the $i$ th type is $e_{i}$, and $c_{i}$, their local concentration, is given by

$$
c_{i}=\frac{n_{i} \exp \left(-e_{i} \psi / k T\right)}{\int \exp \left(-e_{i} \psi / k T\right) d V}
$$

where $n_{i}$ is the number of these ions in $V$. The volume charge density of ions fixed on the polymeric ion $\rho_{p}$ is sometimes replaced by a surface charge density. That is, $\nabla \psi=-4 \pi \rho / D$ is used instead of (1) and a boundary condition relating the potential gradient and the charge per unit area at the surface of the polymeric ion is given. The present approach will apply to both treatments. The subvolume $V$ and the model are chosen with a certain degree of symmetry such that on the boundary of the electrically neutral volume $V$ the potential gradient is zero.

In Eq. (2) the concentration $c_{i}$ and the potential $\psi$ acting on a mobile ion are assumed to be a function only of the three coordinates defining the position of the ion in space, the macro-ion being held fixed in this space. Actually, more rigorously, the potential is a function not only of these three coordinates but also of the instantaneous configuration of the other mobile ions. Similar remarks may be made about the concentration $c_{i} ;$ the local concentration gradients about each mobile ion should be considered explicitly. The assumption made here thus treats the mutual interaction of the 
mobile ions in a more or less approximate manner. ${ }^{7}$ Correspondingly, the Poisson-Boltzmann equation, as used here, will give best results when the mobile ion interacts more with the macro-ion and the many distant mobile ions than with a local mobile ion. This will occur when the mobile ions are dilute, which does not imply that $|e \psi / k T|$ must be small compared with unity.

This viewpoint may also be inferred from the behavior of the activity coefficient of added salt calculated on the basis of Eqs. (1) and (2). At a given polyelectrolyte concentration it tends toward unity as the salt concentration is increased, inasmuch as a given mobile ion is increasingly shielded by the salt from the influence of the macro-ion. In dilute solutions experimental data support this expectation. Nevertheless, for a given polyelectrolyte concentration, as the salt concentration increases the magnitude of the activity coefficient should ultimately approach that observed for the same salt solution containing no polyelectrolyte, rather than unity. This discrepancy is due to the fact that the local concentration gradients in the vicinity of each ion are but approximately taken into account.

(3) Calculations of the electrostatic potential are frequently made for some average configuration of the polymer rather than for all possible ones. This assumes that all important configurations of the polymer have about the same electrostatic free energy arising from the interaction of the charges fixed on the polymer with each other and with the mobile ions in solution.

(4) It will be assumed that the configurational entropy of the polymer chain $S_{p}$ and the average local concentration $C_{p}(\mathbf{r})$ of groups, neutralized plus unneutralized, fixed on the chain depend upon only one quantity, $h$, the configurational variable. This variable may be an average end-to-end distance or the average radius of the polymer, assumed coiled, and its value will depend on the thermodynamic state of the system. It will, for example, increase with increasing charge density along the chain, i.e., with increasing degree of neutralization, and will decrease with increasing shielding of these charges from each other, i.e., with increasing salt concentration. The effect of using a different assumption will be discussed at the end of this section.

In treating this class of models the following contributions to the free energy of a macro-ion and its accompanying mobile ions present in $V$ will be considered :

(a) $S_{p}$, the configurational entropy of the polymer chain, which is related ${ }^{1}$ to the number of configurations of the polymer consistent with a given value of $h$.

(b) $S_{m}$, the entropy of mixing of the charged and uncharged groups along the chain.

(c) $S_{i d}$, the ideal entropy of mixing of the mobile ions.

(d) $F_{e}$, the free energy associated with the reversible charging of the mobile ions and of the macro-ion, during which process the polymer configuration and the number and spatial distribution of ionized groups on the macroion are held fixed. (e) $\sum_{i} n_{i} \mu_{i}{ }^{0}$, where $\mu_{i}{ }^{0}$ is the standard free energy of the mobile ion of the $i$ th kind while $n_{i}$ is the number of such ions in $V$.

(f) $m\left[\alpha \mu_{A}{ }^{0}+(1-\alpha) \mu_{H A}{ }^{0}\right]$, where $\mu_{A}{ }^{0}$ and $\mu_{H A}{ }^{0}$ are the standard free energies of the neutralized and unneutralized groups, respectively, $\alpha$ is the degree of neutralization, and $m$ is the total number of groups on the polymer chain.

The expression for the free energy may therefore be written as

$$
\begin{aligned}
F=-T S_{p}-T S_{m}+F_{e}-T S_{i d} & +\sum_{i} n_{i} \mu_{i}{ }^{0} \\
& +m\left[\alpha \mu_{A}{ }^{0}+(1-\alpha) \mu_{H A}{ }^{0}\right]
\end{aligned}
$$

where $S_{m}$ is given by

$$
S_{m}=-k \int C_{p}\left[\alpha_{r} \ln \alpha_{r}+\left(1-\alpha_{r}\right) \ln \left(1-\alpha_{r}\right)\right] d V
$$

where $C_{p}$ is the local concentration of the groups on the polymer while $\alpha_{r}$ is the local fraction of these groups which are neutralized. Furthermore, $\alpha_{r}$ is related to the charge density $\rho_{p}$ due to ions fixed on the polymer by the relation, $\alpha_{r} C_{p} e_{p}=\rho_{p}$, where $e_{p}$ is the charge of an ion attached to the polymer.

If the simplifying assumption is made that the neutralized and unneutralized groups are randomly mixed then $\alpha_{r}$ becomes independent of position and equal to $\alpha$, the degree of neutralization. Thus, $S_{m}$ becomes

$$
S_{m}=-m k[\alpha \ln \alpha+(1-\alpha) \ln (1-\alpha)] .
$$

While the calculations given below will, in general, be performed without making this assumption, the effect of employing it will be discussed.

Various expressions may be used to estimate the electrostatic contribution $F_{e}$ to the Helmholtz free energy. Neglecting electrostriction the equations given below also represent the corresponding contribution to the Gibbs free energy. $F_{e}$ may be calculated in the following way ${ }^{9}$ from the reversible work to charge the entire system in $V$ at constant configuration and charge distribution of the macro-ion, constant volume, and temperature. The charging process is performed in a manner such that at any stage all ions have the same fraction $\lambda$ of their final charge. The corresponding values of $\psi, \rho, \rho_{p}, c_{i}$, etc. are indicated by the superscript, '. The charge per unit volume, $\rho^{\prime}+\rho_{p}^{\prime}$ ', is $\left(\sum_{i} c_{i}{ }^{\prime} e_{i} \lambda+\alpha_{r} C_{p} e_{p} \lambda\right)$. When the charge on each ion is increased by a fraction $d \lambda$, the charge of those ions in a volume element $d V$ is increased by $\left(\sum_{i} c_{i}{ }^{\prime} e_{i}\right.$ $\left.+\alpha_{r} C_{p} e_{p}\right) d \lambda d V$; that is, by $\left(\rho^{\prime}+\rho_{p}\right)(d \lambda / \lambda) d V$. Since the local value of the potential is $\psi^{\prime}$, an element of work $\psi\left(\rho^{\prime}+\rho_{p}{ }^{\prime}\right)(d \lambda / \lambda) d V$ is done. Summing this work over all ions, i.e., integrating over $V$, the total work done during the complete charging process from $\lambda=0$

\footnotetext{
${ }^{9}$ See E. J. W. Verwey and J. T. G. Overbeek, Theory of Stability of Lyophobic Colloids (Elsevier Publishing Company, Inc., New York, 1948), p. 58.
} 
to $\lambda=1$ is

$$
F_{e}=\int_{\lambda=0}^{\lambda=1} \int_{V} \psi^{\prime}\left(\rho^{\prime}+\rho_{p}{ }^{\prime}\right) \frac{d \lambda}{\lambda} d V=\int_{\lambda} 2 E_{e}{ }^{\prime} \frac{d \lambda}{\lambda}
$$

where $E_{e}{ }^{\prime}$ is the electrostatic energy [see Eq. (7)]. If the polymeric ion had been regarded as forming a surface charge density $\sigma$ over its surface instead of a volume density $\rho_{p}$, then the integral of $\psi^{\prime} \rho_{p}{ }^{\prime}$ over the volume would become an integral over the surface $A$ of the polymer, $\int_{\lambda} \int_{A} \psi^{\prime} \sigma^{\prime}(d \lambda / \lambda) d A$. Similar remarks will apply to all electrostatic expressions throughout this paper. The final equations for the thermodynamic properties will be the same regardless of which picture is used.

A second expression for $F_{e}$ may be obtained from the Gibbs-Helmholtz equation together with an equation for the electrostatic energy, $E_{e}$.

$$
\begin{aligned}
& E_{e}=\frac{1}{2} \int \psi\left(\rho+\rho_{p}\right) d V \\
& F_{e}=T \int_{T=\infty}^{T=T} E_{e} d\left(\frac{1}{T}\right) .
\end{aligned}
$$

The integration in (8) is performed at constant dielectric constant, ${ }^{10}$ and at constant average configuration and charge distribution of the macro-ion. We note that the lower limit arises from the condition that $F_{e} / T=0$ when $T=\infty$.

A third expression for $F_{e}$ may be found by combining the equation for $E_{e}$ with a suitable equation for the entropy of mixing, $S$, of the mobile ions in $V$.

$$
S=-k \sum_{i} \int\left(c_{i} \ln c_{i}-c_{i}\right) d V
$$

where the summation is over all mobile ions. If the total number of ions of type $i$ in the volume $V$ is $n_{i}$, then the ideal entropy of mixing is

$$
S_{i d}=-k \sum_{i}\left(n_{i} \ln \frac{n_{i}}{V}-n_{i}\right)
$$

This equation follows from Eq. (9) when one sets $c_{i}$ equal to its average value $n_{i} / V$. The deviation of the entropy of mixing from its ideal value is $\left(S-S_{i d}\right)$ and

\footnotetext{
${ }^{10} \mathrm{~A}$ few remarks concerning this restriction of integration at constant dielectric constant are perhaps in order. If $E_{e}$ were the total electrostatic contribution $E$ to the energy of the system, then the restriction would be unnecessary, but $E_{e}$ is actually only a portion of $E$. It can be shown that while $\partial\left(F_{e} / T\right) / \partial(1 / T)=E$, $\left[\partial\left(F_{e} / T\right) / \partial(1 / T)\right]_{D}=E_{e}$. Conversely, in order to obtain $F_{e}$ from $E_{e}$ by integration with respect to $1 / T$, it is necessary to hold $D$ constant. The difference between $E$ and $E_{e}$ has been discussed by N. Bjerrum, Z. physik. Chem. 119, 145 (1926). E may be calculated by imagining an isothermal process in which the ions are held motionless throughout and estimating the reversible work done $w_{r}$ by the system and the heat absorbed $q_{r}$ by the system in bringing the ions from infinity to their final equilibrium positions. $w_{r}$ is simply $-E_{e}$ while $q_{r}$ was computed by Bjerrum from the temperature dependence of $w_{r}$ using the second law of thermodynamics to be $-w_{r} \partial \ln D / \partial \ln T$ whence $E=q_{r}-w_{r}=E_{e}(1+\partial \ln D / \partial \ln T)$,
}

we may write as a third expression for $F_{e}$,

$$
F_{e}=E_{e}-T\left(S-S_{i d}\right) \text {. }
$$

In view of the questions which can be raised concerning the internal consistency of solutions of the PoissonBoltzmann equation, it is of interest to examine the extent to which the various expressions for $F_{e}$ are exactly equal when solutions of this equation are introduced into them. In Appendix I it is shown that they are equal.

The third expression for the electrostatic free energy, Eq. (11), is the most convenient one for the present purposes and will be used. Before proceeding it is first observed that Poisson's equation (1) may be written ${ }^{11}$ in the integral form:

$$
\psi(\mathbf{r})=-\frac{1}{D} \int \frac{\rho\left(\mathbf{r}^{\prime \prime}\right)+\rho_{p}\left(\mathbf{r}^{\prime \prime}\right)}{\left|\mathbf{r}-\mathbf{r}^{\prime \prime}\right|} d V .
$$

Equation (12) will be used in some of the proofs given in Appendix I.

In general a thermodynamic function of the system can be expressed in the terms of partial derivatives of the free energy, and a first partial derivative can for example be calculated by estimating the change in free energy corresponding to an appropriate change in the thermodynamic state. The calculation is simplified by observing that in any reversible change of thermodynamic state the contributions to the free energy change arising from a change in configurational variable $h$, a change in the fraction $\alpha$ of the groups on the macroion which are neutralized, and a change in the relative distribution of the charges over the chargeable sites on this polymer are each equal to zero. This circumstance arises since the change in $h, \alpha$, and in the relative distribution of the charges, occurs automatically with the reversible change of state and so contributes nothing to the work done and therefore nothing to the free energy change. The statement concerning $h$ and $\alpha$ is readily established by observing that the equilibrium values of $h$ and $\alpha$ satisfy the equations $(\partial F / \partial h)_{\alpha}=0$ and $(\partial F / \partial \alpha)_{h}=0$, all thermodynamic variables being held constant. Thus the change in free energy corresponding to a reversible change of state equals that calculated at a given $h$ and $\alpha$, for

$$
\delta F=\frac{\partial F}{\partial \alpha} \delta \alpha+\frac{\partial F}{\partial h} \delta h+(\delta F)_{\alpha, h}=(\delta F)_{\alpha, h} .
$$

The statement concerning the relative distribution function, $\alpha_{r} / \alpha$, of charges on the polymer is verified rigorously for the present model in Appendix II. This has the consequence that the free-energy change equals that estimated at fixed $h, \alpha$, and $\alpha_{r} / \alpha$. Equations are also derived in Appendix II for $\alpha_{r} / \alpha$ and for the relation satisfied by the equilibrium value of $h$.

\footnotetext{
${ }^{11}$ E.g., J. C. Slater and N. H. Frank, Introduction to Theoretical Physics (McGraw-Hill Book Company, Inc., New York, 1933), p. 217 .
} 
We shall conclude this section with an amplification of the assumptions (3) and (4) made earlier, and with a brief inquiry into the extent to which the equations deduced here for various thermodynamic properties remain valid when assumption (4) concerning $S_{p}$ and $C_{p}$ is either dropped or replaced by another. First it is observed that for a given polymer molecule many polymer configurations are important and contribute to the configurational entropy $S_{p}$. If these all have substantially the same electrostatic free energy (really, the same $F+T S_{p}$ ), as postulated in assumption (3), then $S_{p}$ will depend only on the same parameter, here $h$, which characterizes $S_{p}$ for the uncharged polymer. That is, assumptions (3) and (4) are not mutually inconsistent. If, further, many of these configurations are substantially different so that appreciable fluctuations of the configuration of a polymer molecule from the average configurational distribution function, $C_{p}(\mathbf{r})$, occurs in time, then it is the parameter $h$, rather than the function $C_{p}(\mathbf{r})$ which maximizes the free energy. That is, in the expression for $\delta F$, the coefficient of $\delta h$ rather than of $\delta C_{p}$ is set equal to zero at equilibrium.

Consider now a different model, one in which all the important configurations of a polymeric molecule are very similar so that only small fluctuations from the distribution function $C_{p}(\mathbf{r})$ occur. It is expected that these configurations will have substantially the same electrostatic free energy, and assumption (3) thus remains valid. In this model $C_{p}$ is calculated by maximizing the free energy. That is, $C_{p}$ describes that distribution of groups on the polymer which maximizes the free energy. This has the immediate consequence that in a reversible change of state this spatial distribution function of groups on a polymer will automatically adjust itself and, like the redistribution of the charges on these groups, contribute nothing to $\delta F$. This may be verified by writing, in contrast to the previous model where $S_{p}$ and $C_{p}$ were only indirectly related (through $h$ ), for this case $S_{p}=\int g\left(C_{p}\right) d V$, where $g$ is a function of $C_{p}$ alone and introducing this into Eq. (56) for the free energy in Appendix II. The procedure used there is then followed except that the variation of the free energy is computed in terms of $\delta C_{p}$ instead of $\delta h$. As before $\partial F / \partial \alpha=0$ and it is concluded that in this alternative model the free-energy change equals that estimated at fixed $\alpha, \alpha_{r} / \alpha$ and $C_{p}$. These restrictions are equivalent to constant $\alpha, \alpha_{r} / \alpha$, and $h$, since when $h$ is constant, $C_{p}$ is constant. Thus, the various equations deduced below for the thermodynamic properties remain valid if this alternative model of polyelectrolytes be assumed.

\section{Calculation of Activity Coefficients}

The chemical potential, and hence the activity coefficient, of added salt may be calculated by differentiating the sum of all contributions to the free energy with respect to amount of salt. According to the discussion in the previous section, only the derivative at constant degree of neutralization, at constant polymer configuration, and fixed distribution of polymer charges need be calculated. When these variables are held constant $S_{p}$ and $S_{m}$ do not change and, as seen from Eq. (3), it is sufficient to calculate the changes in $F_{e},-T S_{i d}$ and $\sum_{i} n_{i} \mu_{i}{ }^{0}$.

Inasmuch as $\left(F_{e}-T S_{i d}\right)$ equals $\left(E_{e}-T S\right)$, Eq. (11), the changes in $E_{e}$ and $S$ will be determined rather than the changes in $F_{e}$ and $S_{i d}$. According to $\mathrm{Eq}$. (7) the change in $E_{e}$ with amount of added salt at constant volume $V$ may be written as

$$
\begin{aligned}
\delta E_{e} & =\frac{1}{2} \int\left[\psi\left(\delta \rho+\delta \rho_{p}\right)+\left(\rho+\rho_{p}\right) \delta \psi\right] d V \\
& =\int\left(\rho+\rho_{p}\right) \delta \psi d V=\int \psi\left(\delta \rho+\delta \rho_{p}\right) d V .
\end{aligned}
$$

The second equation may be established by writing $\psi$ and $\delta \psi$ in terms of the $\rho$ 's and $\delta \rho$ 's using Eq. (12). At constant polymer configuration and distribution of polymer charges $\delta \rho_{p}=0$ so that

$$
\delta E_{e}=\int \psi \delta \rho d V
$$

The change in $S$ at constant volume may be estimated from (9) to be

$$
\delta S=-k \sum_{i} \int \ln c_{i} \delta c_{i} d V .
$$

Using the Boltzmann expression (2) and denoting $\int \exp \left(-e_{i} \psi / k T\right) d V$ by $V_{i}$, it follows that

$$
\begin{aligned}
\delta S & =-k \sum_{i} \int\left(-\frac{e_{i} \psi}{k T}+\ln \frac{n_{i}}{V_{i}}\right) \delta c_{i} d V \\
& =\frac{1}{T} \int \psi \delta \rho d V-k \sum_{i}\left(\ln \frac{n_{i}}{V_{i}}\right) \int \delta c_{i} d V
\end{aligned}
$$

since $\delta \rho=\sum_{i} e_{i} \delta c_{i}$. It is noted that $n_{i} / V_{i}$ is independent of position and hence has been removed from the second integral.

At constant volume $\delta n_{i}=\int \delta c_{i} d V$ so that from Eqs. (14) and (17) we have

$$
\delta\left(E_{e}-T S\right)=k T \sum_{i} \ln \frac{n_{i}}{V_{i}} \delta n_{i}
$$

The change in the term $\sum_{i} n_{i} \mu_{i}{ }^{0}$ caused by the addition of salt is $\sum_{i} \mu_{i}{ }^{0} \delta n_{i}$.

Thus the chemical potential of any mobile ion of the type $j$ in $V$ is

$$
\mu_{j}=\frac{\partial}{\partial n_{j}}\left(E_{e}-T S+\sum_{i} n_{i} \mu_{i}{ }^{0}\right)=\mu_{j}{ }^{0}+k T \ln \frac{n_{j}}{V_{j}} .
$$


The chemical potential of any salt, one mole of which dissociates into $\nu_{+}$moles of cations and $\nu_{-}$moles of anions in this sytem, is therefore

$$
\mu=\nu_{+} \mu_{+}^{0}+\nu_{-} \mu_{-}^{0}+k T \ln \left(\frac{n_{+}}{V_{+}}\right)^{\nu_{+}}\left(\frac{n_{-}}{V_{-}}\right)^{\nu_{-}}
$$

where the subscripts + and - correspond to the cations and anions, respectively.

Defining the mean ion activity coefficient $f_{ \pm}$by the equation

$$
\mu=\nu_{+} \mu_{+}{ }^{0}+\nu_{-} \mu_{-}{ }^{0}+k T \ln \left(\frac{n_{+}}{V}\right)^{\nu_{+}}\left(\frac{n_{-}}{V}\right)^{\nu_{-}} f_{ \pm}{ }^{\nu}
$$

where $\nu=\nu_{+}+\nu_{-}$, it follows that

$$
f_{ \pm}^{\nu}=\left(\frac{V}{V_{+}}\right)^{\nu_{+}}\left(\frac{V}{V_{-}}\right)^{\nu}
$$

It may be verified that making an additional assumption of random mixing of neutralized and unneutralized groups on the polymer, i.e., setting $\alpha_{r}=\alpha$ everywhere and so using Eq. (5) for $S_{m}$ instead of (4), leaves Eq. (22) unaltered.

It is interesting to observe that this equation can also be obtained in a somewhat different, though less rigorous, way. Let us assume that this salt-polyelectrolyte solution is in equilibrium with an ideal salt solution containing no polyelectrolyte, where the concentration of ions of the $i$ th type is $\bar{c}_{i}$, the two phases being separated by a membrane impermeable to the macro-ion in order to maintain equilibrium. That is, a type of Donnan membrane equilibrium is set up. Then it is reasonable to expect that the concentration of ions of type $i$ in any local region of the subvolume $V$ in the polyelectrolyte phase will be given by $c_{i}=\bar{c}_{i} \exp \left(-e_{i} \psi / k T\right)$ since $c_{i}=\bar{c}_{i}$ in the ideal solution where $\psi=0$. Thus $n_{i}=\bar{c}_{i} \int \exp \left(-e_{i} \psi / k T\right) d V$. At equilibrium the activity of a salt is the same in both phases. That is,

$$
f_{ \pm}{ }^{\nu}\left(n_{+} / V\right)^{\nu+}\left(n_{-} / V\right)^{\nu_{-}}=\left(\bar{c}_{+}\right)^{\nu_{+}}\left(\bar{c}_{-}\right)^{\nu_{-}} \text {. }
$$

Since, as just stated, $n_{i}=\bar{c}_{i} V_{i}$, Eq. (22) immediately follows.

That the mean ion activity coefficient, calculated on this basis, is always equal to or less than unity when both ions of the salt have the same valence, may be shown by application of Schwarz's inequality ${ }^{12}$ : $\int f^{2} d V \cdot \int g^{2} d V \geqslant\left(\int f g d V\right)^{2}$. Setting $f=\exp \left(-e_{+} \psi / 2 k T\right)$ and $g=\exp (-e, \psi / 2 k T)$ it follows that $f g=1$ when $e_{+}=-e_{-}$. The integral on the right-hand side of the inequality then becomes $V^{2}$ while the product on the left-hand side is $V_{+} V_{-}$. That is, $V_{+} V_{-} \geqslant V^{2}$ and, since $\nu_{+}=\nu_{-}$here, $f_{ \pm}$is seen from Eq. (22) to be equal to or less than unity.

12 H. Margenau and G. M. Murphy, The Mathematics of Physics and Chemistry (D. Van Nostrand Company, Inc., New York, 1943), p. 130.
A few applications, considered below, may serve to indicate the ease of a calculation based on Eq. (22) as compared with one involving a differentiation of the free energy into which parameters of the models have been previously introduced.

\section{Applications}

A coiled model of polyelectrolytes.-The PoissonBoltzmann equation has been solved ${ }^{7}$ for a spherically symmetrical model of a polyelectrolyte, using the approximation that a large number of mobile ions lie within the coils such that this region is about electrically neutral. The result obtained by these authors for the chemical potential of added salt [their Eqs. (32) and (33)] may also be obtained in a very straightforward manner by using Eq. (22) here and their Eqs. (23) and (24). This procedure avoids an awkward integration and differentiation. Actually these two values for $\mu$ differ roughly by an additive constant due to a slight difference in the definition of the entropy..$^{13}$

"Parallel rod" model of polyelectrolyies.-Recently the Poisson-Boltzmann equation has been solved ${ }^{3,4}$ exactly for the cylindrically symmetric case, no added salt, in which the polymeric ion assumed to be rod-like lies along the axis. No exact solution for $\psi$ is available for the important case of added salt. Nevertheless the solution of these authors can also be applied to the calculation of the activity coefficient of infinitesimal amounts of such salt, inasmuch as such minute traces do not affect $\psi$. These authors, however, did not concern themselves with added salt and did not calculate any activity coefficient. Using their expression for $\psi[\mathrm{Eq}$. (33) of reference 4$]$, the product $V_{+} V_{-}$was calculated and it is found after considerable simplification that for a uni-univalent salt

$$
f_{ \pm}^{2}=2 \frac{1-(a / R)^{2}}{\lambda^{*}\left[f_{1}-(a / R)^{4} f_{2}\right]}
$$

where $a=$ radius of the rod, $R=$ radius of the cylinder $\left(\pi R^{2}\right.$ is the volume of solution per macro-ion per unit length of the macro-ion), $\lambda^{*}=e^{2} \nu^{*} / D k T h^{*}$, there being $\nu^{*}$ ions fixed on a macro-ion of length $h^{*}, \beta$ is the solution of $\lambda^{*}=\left(1+\beta^{2}\right) /\{1-\beta \cot [\beta \ln (a / R)]\}$ and $f_{1}$ and $f_{2}$ are functions of $\beta$. Thus

$$
f_{1}=\frac{13+\beta^{2}}{\left(4+\beta^{2}\right)\left(1+\beta^{2}\right)}, \quad f_{2}=\frac{\left(\lambda^{*}-3\right)^{2}+4+\beta^{2}}{\left(4+\beta^{2}\right)\left[\left(\lambda^{*}-1\right)^{2}+\beta^{2}\right]} .
$$

Depending on the magnitudes of the parameters $\lambda^{*}$ and $a / R, \beta$ may become imaginary. When $\beta$ becomes

\footnotetext{
${ }^{13}$ Osawa $e t$ al. use a definition of the entropy of mixing based on mole fractions while here a definition based on concentrations is used. The latter is more simply related to the definitions of $E_{e}$ and $F_{e}$ while the former is more exact but also more awkward. In dilute solution they differ by an additive constant. In very concentrated solutions where a significant difference occurs neither presumably is especially correct, the effect of hydration and restricted volume on this entropy becoming very important then.
} 


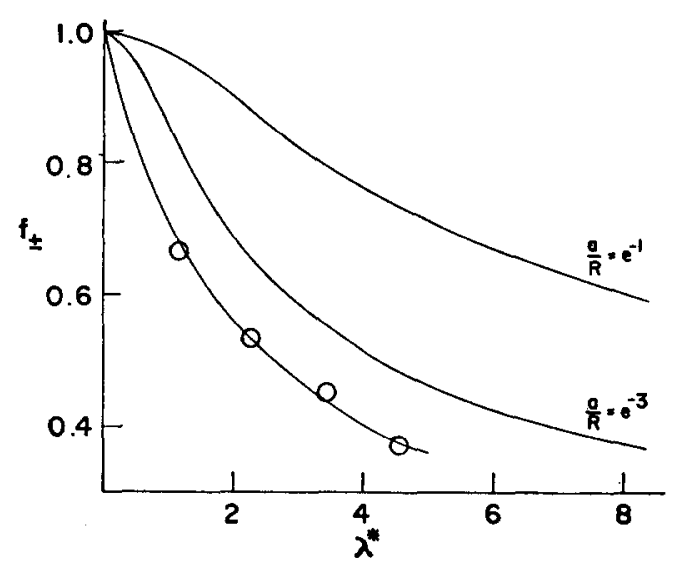

FIG. 1. The dependence of the activity coefficient $f_{ \pm}$of vanishngly small amounts of added salt on the charge parameter $\lambda^{*}$ of the polyelectrolyte: theoretical, Calc for $a / R=e^{-1}$ and for $a / R=e^{-8}$; experimental, $\ominus$ data of $f_{ \pm} v s \alpha$ are plotted on this graph using $\lambda^{*}=5.9 \alpha$ as the conversion factor (see text).

imaginary it should be replaced in all these equations by $i|\beta|$ and we observe that

$$
\cot (i|\beta| \ln a / R)=-i \operatorname{coth}(|\beta| \ln a / R) .
$$

Reasonable estimates of $\lambda^{*}$ and of $a / R$ may be made although some uncertainty arises because of the kinked nature of the polymeric ion. As the degree of neutralization $\alpha$ of polymethacrylic acid, say, increases, the polymeric ion becomes more highly charged and stretches due to the repulsion of its charges. When $\alpha$ exceeds about 0.35 viscosity and light scattering measurements ${ }^{14}$ indicate that the dimensions change relatively little with increasing $\alpha$. The viscosity data ${ }^{14}$ suggest that for these values of $\alpha$ the head-to-tail length of the molecule is about half that of the linear chain length; that is, half the value it would have were it fully stretched. The question arises as to which length to use for $h^{*}$. The effective surface charge density acting on mobile ions at appreciable distances from the macro-ion should be that corresponding to the value estimated from observed head-to-tail length while the effective density acting on mobile ions very near the macro-ion might approximately be that estimated from the total linear chain length.

This uncertainty in $h$ introduces a corresponding one in $a / R$ and $\lambda^{*}$. The calculated values of $f_{ \pm}$are relatively insensitive to such errors in $a / R$ while the effect of $f_{ \pm}$ of an uncertainty in $\lambda^{*}$ of a factor of 2 may be inferred from Fig. 1. In the present calculation we shall use the observed head-to-tail length, so that $h / \nu^{*}=1.25 \mathrm{~A}$. When the concentration of polyelectrolyte is $c_{0}$ equivalents per liter, then $\pi R^{2} h^{*} c_{0}=\nu^{*}$, so that when $R$ and $h$ are in $\mathrm{A}$, we find $R=20.6 / \sqrt{ } c_{0} \mathrm{~A}$. When $c_{0}=0.05 \mathrm{M}$, as in the following data, when $R=92 \mathrm{~A}$. The radius, $a$, of the cylindrical, charged rod is essentially the distance of closest approach of the mobile ions to a carboxyl ion fixed on the polymer chain. If $a$ is about $6 \mathrm{~A}$, then

\footnotetext{
${ }^{14}$ See A. Oth and P. Doty, J. Phys. Chem. 56, 43 (1952).
}

$a / R=e^{-2.75}$. Other reasonable choices of $a$ will have only a minor effect on the calculated values of the activity coefficient. When $\alpha$ exceeds 0.35 we estimate from the previously mentioned head-to-tail length $\lambda^{*}=5.7 \alpha$, approximately.

In Fig. 1 the mean ion activity coefficients of traces of salt in the presence of polyelectrolyte, estimated from Eq. (23), are given as a function of $\lambda^{*}$ for $a / R=e^{-1}$ and $a / R=e^{-3}$. In performing these calculations, $\lambda^{*}$ was calculated for various values of $\beta$, for these two values of $a / R$, using the equation relating $\lambda^{*}$ to $\beta$, rather than solving this transcendental equation for $\beta$. A graphical representation for the dependence of $\lambda^{*}$ on $\beta$ has been given by Lifson and Katchalsky. ${ }^{15}$

Experimental activity coefficients ${ }^{16}$ have been determined for various amounts of added salt as a function of the degree of neutralization. These data extrapolated to zero concentration of added salt are plotted in the form $f_{ \pm}$vs $\lambda^{*}$ in Fig. 1 assuming $\lambda^{*}=5.7 \alpha$. The agreement with the curve for $a / R=e^{-3}$ is reasonably good considering the nature of the approximations and the fact that no adjustable parameters were used. To obtain exact agreement an arbitrary choice of $\lambda^{*}=9.5 \alpha$ is necessary.

\section{Calculation of Osmotic Pressure}

The osmotic pressure of a salt-polyelectrolyte system may be calculated by differentiating the free energy, as given by Eq. (3), with respect to volume keeping the amounts of polyelectrolyte and added salt constant. Recalling from the discussion in Sec. 1 that this derivative equals that at constant polymer configurational variable $h$, fixed polymer charge distribution and fixed degree of neutralization, the contributions to the free energy which may change with volume under these restrictions are $-T S_{m}, E_{e}$ and $-T S$ as defined in Eqs. (4), (7), and (9), respectively. The changes in these quantities will now be calculated. The contribution of the motion of the macro-ion to the osmotic pressure will, however, be neglected for the present purposes.

A change in volume $\delta V$ will change the potential, the charge density and the upper limit of integration of the expressions for $S_{m}, E_{e}$, and for $S$. The contribution to $\delta S_{m}$ arising from a change in the upper limit of integration in (4) is zero since the polymer lies within $V$ rather than on the bounding surface $S$ of this volume. At fixed distribution of polymer charges, $\alpha_{r}$ does not change so that the change in the integrand of the expression for $S_{m}$ is also zero. That is, $\delta S_{m}$ is zero.

Indicating the values of $\psi$ and $\rho$ on the surface of the volume $V$ by a superscript $s$ we have

$$
\begin{aligned}
2 \delta E_{e}=\int \psi(\delta \rho+ & \left.\delta \rho_{p}\right) d V \\
& +\int\left(\rho+\rho_{p}\right) \delta \psi d V+\psi^{s}\left(\rho^{s}+\rho_{p}{ }^{s}\right) \delta V
\end{aligned}
$$

\footnotetext{
${ }^{15}$ S. Lifson and A. Katchalsky, J. Polymer Sci. 13, 43 (1954).
}

${ }^{16}$ A. Katchalsky and S. Lifson, J. Polymer Sci. 11, 409 (1953). 
By expressing $\delta \psi$ and $\psi$ in terms of the $\rho$ 's using Eq. (12), it is found that

$$
\begin{aligned}
\delta E_{e} & =\int\left(\rho+\rho_{p}\right) \delta \psi d V \\
& =\int \psi\left(\delta \rho+\delta \rho_{p}\right) d V+\psi^{s}\left(\rho^{s}+\rho_{p}^{s}\right) \delta V .
\end{aligned}
$$

At fixed polymer configuration and polymer charge distribution $\delta \rho_{p}=0$ and since $\rho_{p}$ lies within $V$ rather than on its surface $S, \rho_{p}{ }^{s}=0$. Thus it follows that

$$
\delta E_{e}=\int \psi \delta \rho d V+\psi^{s} \rho^{8} \delta V
$$

We also have from (9)

$$
\delta S=-k \sum_{i}\left(c_{i}{ }^{8} \ln c_{i}{ }^{s}-c_{i}{ }^{s}\right)-k \sum_{i} \int \ln c_{i} \delta c_{i} d V
$$

Using the Boltzmann expression for $c_{i}$, we obtain

$$
\begin{aligned}
T \delta S=\rho^{s} \psi^{8} \delta V-k T \sum_{i}( & \left.c_{i}{ }^{\delta} \delta V+\int \delta c_{i} d V\right) \ln \frac{n_{i}}{V_{i}} \\
& +\int \psi \delta \rho d V+k T \sum_{i} c_{i}{ }^{s} \delta V .
\end{aligned}
$$

The change in the number of mobile ions of the $i$ th type $\delta n_{i}$ is equal to $\left(c_{i}{ }^{s} \delta V+\int \delta c_{i} d V\right)$ and is zero. It thus follows that

$$
\delta F=\delta E_{\dot{e}}-T \delta S=-k T \sum_{i} c_{i}{ }^{8} \delta V .
$$

The introduction of solvent to increase the volume also results in another free-energy change which in dilute solution becomes $\mu_{w}{ }^{0} \delta n_{w}$ to a good approximation, $\mu_{w}{ }^{0}$ being the chemical potential of pure solvent. Inclusion of this in (30) and division of the equation by $\delta n_{w}$ yields for the chemical potential of the solvent $\mu_{w}=\mu_{w}{ }^{0}$ $+(\partial F / \partial V) \bar{V}_{w}, \bar{V}_{w}$ being the partial molar volume of solvent. Inasmuch as the osmotic pressure $\pi$ equals $\left(\mu_{w}{ }^{0}-\mu_{w}\right) / \bar{V}_{w}$ in dilute solutions, we have

$$
\pi=-\frac{\partial F}{\partial V}=k T \sum_{i} c_{i}{ }^{s} .
$$

It may be verified that the additional assumption of random mixing of the neutralized and unneutralized groups on the polymer chain leads to the same equation for $\pi$.

The result (31) for the contribution of the mobile ions is quite reasonable. The potential gradient at the boundary of the volume $V$ is zero so that the ions there are, according to the present formalism, not acted on by any electrostatic forces. That is, the solution at the boundary is an "ideal solution," and the osmotic pressure of an ideal solution is $k T$ multiplied by the total concentration of its solute. ${ }^{17}$ Since the concentration at the boundary is $\sum_{i} c_{i}{ }^{s}$ the osmotic pressure is $k T \sum_{i} c_{i}{ }^{3}$. This sum is, at least in the absence of added salt, less than the average total concentration in the solution so that the osmotic pressure is less than the value it would have if the solution were everywhere ideal. When the solution becomes ideal the concentration of an ion at the boundary becomes equal to its average concentration, $n_{i} / V$, and the osmotic pressure assumes its ideal value $\sum_{i}\left(n_{i} / V\right) k T$.

As an application of (31), we consider the derivation of an expression obtained for the osmotic pressure using the "parallel rod" picture. By computing the electrostatic free energy in terms of the parameters, $a / R$ and $\lambda^{*}$, of this model and differentiating it with respect to the volume, Katchalsky and Lifson ${ }^{15}$ calculated the osmotic pressure. The differentiation is, however, a particularly tedious one and an equation for the osmotic pressure can be obtained much more simply using (31) and their expression for the concentration. Thus we find immediately

$$
\pi=\nu^{*} k T\left(1-\beta^{2}\right) n_{p} / 2 \lambda^{*}
$$

where $n_{p}=1 / \pi R^{2} h^{*}$, the number of macro-ions per cc, and $\beta, \nu^{*}$, and $\lambda^{*}$ have been defined earlier. Actually this equation is slightly different from Eq. (34) of their paper. This difference arises from a minor error in the definition there of the osmotic pressure of the uncharged system, $\pi_{i}$ [their Eq. (30)] which does not take into account the volume of the solution unavailable to the mobile ions. Their value of $\pi_{i}$ should be multiplied by the factor $R^{2} /\left(R^{2}-a^{2}\right)$.

Finally it is to be observed that Eq. (31) is independent of Eq. (22) which was derived in the previous section. These equations are related to the chemical potentials of solvent and added salt, respectively, and these are independent. However, any expression derived for the chemical potential of the polyelectrolyte would not be independent of these since the GibbsDuhem equation connects all three chemical potentials.

\section{Calculation of Titration Curves}

It will be assumed for simplicity in this section that the protons attached to some of the basic groups in these polybasic acids (or bases) are randomly distributed among all groups. That is, Eq. (5) for $S_{m}$ will be used rather than Eq. (4). This may be regarded as a type of Bragg-Williams approximation used in the treatment of order-disorder phenomena. The error inherent in this assumption for the case where nearest neighbor interaction between the charged groups of polyelectrolytes predominates has been considered elsewhere. ${ }^{18}$ Under the experimental conditions discussed there (appreciable added salt present) it appeared to be a fairly good approximation. In the present

${ }_{17}$ This argument was suggested to the writer by Dr. $H$. Morawetz.

${ }_{18}$ R. A. Marcus, J. Phys. Chem. 58, 621 (1954). 
model the assumption will be the more appropriate the less $\psi$ (and therefore, according to $(65), \alpha_{r}$ ) varies over the space occupied by the macro-ion.

It will be recalled that the equilibrium value of the degree of neutralization $\alpha$ is determined by setting the variation in the free energy equal to zero, the volume, salt, number of available protons, and polymer configuration being held fixed. The change in the various contributions to $F$, listed in Eq. (3), will now be estimated. At constant polymer configuration $S_{p}$ is constant. The term $\left(F_{e}-T S_{i d}\right)$ equals $\left(E_{e}-T S\right)$. At constant volume, $\delta E_{e}$ and $\delta S$ are given by Eqs. (14) and (17), respectively. With the added restriction of constant salt concentration it thus follows that

$$
\delta\left(E_{\varepsilon}-T S\right)=\int \psi \delta \rho_{p} d V+k T \ln \frac{n_{H}}{V_{H}} \delta n_{H} .
$$

At constant polymer configuration and assumed random distribution of neutralized and unneutralized groups $\rho_{p}$ changes only because of a change in the total number of ionized groups. Since the ionized groups are assumed to be randomly distributed the local concentration of the ionized groups on the polymer is proportional to the sum of the concentrations of all groups, neutralized plus unneutralized. Therefore the fractional change in the charge density $\delta \rho_{p} / \rho_{p}$ with change in $\alpha$ is constant throughout the polymer and is, in fact, equal to the over-all fractional change in the number of ionized groups, $\delta \alpha / \alpha$. That is, $\delta \rho_{p}$ in Eq. (33) is related to $\delta \alpha$ according to the equation

$$
\frac{\delta \rho_{p}}{\rho_{p}}=\frac{\delta \alpha}{\alpha}
$$

The change in $S_{m}$, given by (5) is

$$
\delta S_{m}=-m k[\ln (\alpha / 1-\alpha)] \delta \alpha,
$$

while the variation in $\sum_{i} n_{i} \mu_{i}{ }^{0}$ at constant salt is $\mu_{H}{ }^{0} \delta n_{H}$. Observing that $\int \delta \rho_{p} d V=e_{p} m \delta \alpha=-e_{H} \delta n_{H}$ it then follows, if the individual ions on the polymer are univalent anions, say, $\left(e_{p}=-e_{H}\right)$, that

$$
\begin{aligned}
& \delta F=\left[k T \ln \frac{\alpha}{1-\alpha}+\left(\mu_{H}^{0}+\mu_{A}^{0}-\mu_{H A}{ }^{0}\right)\right. \int \psi \delta \rho_{p} d V \\
&\left.+\frac{\int \alpha}{m \alpha}+k T \ln \frac{n_{H}}{V_{H}}\right] \delta n_{H}
\end{aligned}
$$

i.e.,

$$
-\ln \frac{\alpha n_{H}}{(1-\alpha) V_{H}}=\frac{\Delta \mu^{0}}{k T}+\frac{\int \psi \rho_{p} d V}{m \alpha k T} .
$$

Equation (36) may be used, in conjunction with Eq. (22) for the mean ion-activity coefficient, to estimate the activity of some acid such as $\mathrm{HCl}$ in the presence of a polymeric acid as a function of the latter's degree of neutralization, $\alpha$. For example, the logarithm of the activity of $\mathrm{HCl}$ is

$$
\begin{aligned}
\ln a_{H C l}=\ln \frac{n_{H} n_{C l} f_{ \pm}{ }^{2}}{V_{H} V_{C l}} & \\
& =-\frac{\Delta \mu^{0}}{k T}-\frac{\int \psi \rho_{p} d V}{m \alpha k T}+\ln \left[\frac{(1-\alpha) n_{C l}}{\alpha V_{C l}}\right]
\end{aligned}
$$

where $V_{C l}=\int \exp (e \psi / k T) d V$.

Alternatively, Eq. (36) may be used to obtain an expression for the dependence of $p \mathrm{H}$ on $\alpha$ but there is some uncertainty arising from the liquid junction potential, $E_{L}$, present in the electrochemical cell used to define the $p \mathrm{H}$. If in the presence of salt $E_{L}$ is reasonably independent of the degree of neutralization, $\alpha$, of the polymeric acid or base, then a calculation of the change of $p \mathrm{H}$ with $\alpha$ only involves an estimate of the change in $-\log a_{H}$ with $\alpha$, for then the $p \mathrm{H}=-\log a_{H}$ t constant. According to Eq. (19) previously given for the chemical potential of an ion, $-\log a_{H}=-\log n_{H} / V_{H}$ and it therefore follows from (36) that

$$
p \mathrm{H}=\log \frac{\alpha}{1-\alpha}+\frac{\Delta \mu^{0}}{2.3 k T}+\frac{\int \psi \rho_{p} d V}{2.3 m \alpha k T}+\text { constant. }
$$

Defining the $p K$ of the polymeric acid as $p \mathrm{H}-\log \alpha / 1-\alpha$ we have to the same degree of approximation

$$
p K=\frac{\Delta \mu^{0}}{2.3 k T}+\frac{\int \psi \rho_{p} d V}{2.3 m \alpha k T}+\text { constant }
$$

When the ions fixed on the polymer chain are regarded as forming a surface charge density $\sigma$ rather than a volume charge density $\rho_{p}$, the integral in (38) and (39) should be replaced by the surface integral $\int_{A} \psi \sigma d A$.

When no added salt is present, it is possible that depending upon the conditions the change of ionic concentration accompanying neutralization may have a somewhat larger effect on the liquid junction potential than is the case when salt is present. However, in the absence of added salt the repulsion of the like charges on a macro-ion is usually poorly shielded and the change of $p \mathrm{~K}$ with $\alpha$ is generally quite large. Changes in $E_{L}$ with $\alpha$ are presumably of a smaller order of magnitude. In that case Eq. (39) may again be used.

If in this treatment the simplifying assumption of random mixing of neutralized and unneutralized groups had not been made, then an equation similar to (38) would have been obtained, but with the exception that $\left(\int \psi \rho_{p} d V\right) /(m \alpha)+k T \ln (\alpha / 1-\alpha)$ would have been replaced by $k T \ln D$ where $D$ is defined in (66). 


\section{Note on the Equilibrium Configuration}

In the calculation of the various thermodynamic properties a knowledge of the polymer configuration is necessary. Making certain assumptions, a parameter roughly defining this configuration may be estimated from viscosity or light scattering measurements, say. This parameter may, for example, be the radius of the polyelectrolyte, assumed to be coiled. If, instead, the polymer is assumed to be rod-like no such additional information is necessary.

On the other hand, the equilibrium value of a configurational variable such as radius of polymer or average end-to-end distance may be calculated by setting the change in the free energy $F$ equal to zero for any deviation of this variable from its equilibrium value, at constant composition, volume, temperature, and degree of neutralization. In Appendix II it has been shown in this manner that the equilibrium value of $h$ satisfies the relation

$$
k T \int \ln \left(1-\alpha_{r}\right) \frac{d C_{p}}{d h} d V=T \frac{d S_{p}}{d h} .
$$

A knowledge of the dependence of $\alpha_{r}$ and $\psi$ on position and on $h$, and of $C_{p}$ and $S_{p}$ on $h$, is needed to solve this equation. Thus $\alpha_{r}$ is related to $\psi$ by the relation (65), $\alpha_{r}=D e^{-e_{p} \psi / k T} /\left(1+D e^{-e_{p} \psi / k T}\right)$, which can be introduced into the Poisson-Boltzmann equation. This latter equation may then be solved for $\psi(r, h)$. Estimates of the dependence of $C_{p}$ and $S_{p}$ on $h$ for the uncharged polymer can be made and, according to assumption (3), of the first section, these could be used for the macro-ion in order to solve Eq. (40) for $h$.

When random mixing of the neutralized and unneutralized groups on the polymetric molecule is assumed, then it may be verified by using the same procedure as in Appendix II, only setting $\alpha_{r}=\alpha$ in Eq. (56) for the free energy there, that $h$ is the solution of

$$
\alpha e_{p} \int \psi \frac{d C_{p}}{d h} d V=T \frac{d S_{p}}{d h} .
$$

Alternatively, jt can readily be shown with the aid of (65) that (40) reduces to this equation when $\psi$ varies but little over the space occupied by the polymer.

\section{ACKNOWLEDGMENTS}

The writer would like to express his appreciation to Dr. E. M. Loebl and Dr. H. Morawetz of this institute who read the manuscript critically and offered a number of helpful comments.

\section{APPENDIX I}

\section{Comparison of Various Expressions for Fe}

(i) Comparison of Eqs. (6) and (8).

When the degree of charging of the entire system is $\lambda$ the corresponding value of the electrostatic free energy will be denoted by $F_{e}{ }^{\prime}$. That is, $F_{e}{ }^{\prime}=F_{e}$ when $\lambda=1$. Accordingly, we may rewrite these equations in terms of $F_{e}^{\prime}$ :

$$
\begin{aligned}
& F_{e}{ }^{\prime}=\int_{\lambda=0}^{\lambda=1} 2 E_{e}^{\prime} \frac{d \lambda}{\lambda}, \\
& F_{e}{ }^{\prime}=T \int_{T=\infty}^{T=T} E_{e} d\left(\frac{1}{T}\right),
\end{aligned}
$$

where the integration in $\left(8^{\prime}\right)$ is to be performed at constant dielectric constant $D$. The superscript ' will be used to signify the values of $\psi, \rho$, etc. when $\lambda \neq 1$. Differentiating the first expression with respect to $\lambda$ we have

and therefore

$$
\left(\frac{\partial F_{e}^{\prime}}{\partial \lambda}\right)_{T}=\frac{2 E_{e}{ }^{\prime}}{\lambda}
$$

$$
\left(\frac{\partial\left(F_{e}^{\prime} / T\right)}{\partial \lambda}\right)_{T}=\frac{2 E_{e}{ }^{\prime}}{\lambda T} .
$$

Deriving the second with respect to $1 / T$ at constant $D$ we have

$$
\left(\frac{\partial\left(F_{e}^{\prime} / T\right)}{\partial(1 / T)}\right)_{\lambda, D}=E_{e}{ }^{\prime}
$$

To show the equivalence of Eqs. (6) and (8) it is sufficient, therefore, to show that

$$
\left(\frac{\partial\left(F_{e}^{\prime} / T\right)}{\partial \lambda}\right)_{T}=\frac{2}{\lambda T}\left(\frac{\partial\left(F_{e}^{\prime} / T\right)}{\partial(1 / T)}\right)_{\lambda, D} .
$$

If at constant $D, F_{e}^{\prime}$ depends on $\lambda$ and $T$ only through a variable $\lambda^{2} / T$, then this equation can readily be derived by expressing each partial in terms of the derivative with respect to $\lambda^{2} / T$ :

$$
\begin{array}{r}
\left(\frac{\partial F_{e}^{\prime} / T}{\partial \lambda}\right)_{T}=\left(\frac{\partial F_{e}^{\prime} / T}{\partial \lambda^{2} / T}\right)_{D}\left(\frac{\partial \lambda^{2} / T}{\partial \lambda}\right)_{T} \\
=\frac{2 \lambda}{T} \frac{\partial F_{e}^{\prime} / T}{\partial \lambda^{2} / T} \\
\left(\frac{\partial F_{e}^{\prime} / T}{\partial 1 / T}\right)_{\lambda, D}=\left(\frac{\partial F_{e}^{\prime} / T}{\partial \lambda^{2} / T}\right)_{D}\left(\frac{\partial \lambda^{2} / T}{\partial 1 / T}\right)_{\lambda} \begin{array}{r}
\partial F_{e}^{\prime} / T \\
\partial \lambda^{2} / T
\end{array}
\end{array}
$$

The elimination of $\partial F_{e}^{\prime} / T / \partial \lambda^{2} / T$ from these equations yields the desired equation. As expected, at constant $D$ the statistical mechanical expression for $F_{e}{ }^{\prime} / T$,

$$
\begin{array}{r}
F_{e}^{\prime}=-k T \ln \left[\int \cdots \int \exp \left(-\sum_{i>i} \frac{\lambda^{2} e_{i} e_{j}}{\left|\mathbf{r}_{i}-\mathbf{r}_{j}\right| D k T}\right)\right. \\
\left.\times d V_{1} \cdots d V_{N} / V^{N}\right]
\end{array}
$$


depends on $\lambda$ and $T$ only through a dependence on $\lambda^{2} / T$ and so fulfills this equation. The integration is over the coordinates of all mobile ions in $V$ but the $i$ and $j$ refer to the coordinates of all ions.

The Poisson-Boltzmann equation can be shown to yield the same dependence of $F_{e}^{\prime} / T$ on $\lambda$ and $T$. We first observe that

$$
\psi^{\prime}(\mathbf{r})=-\frac{1}{D} \int \frac{\rho^{\prime}\left(\mathbf{r}^{\prime \prime}\right)+\rho_{p}{ }^{\prime}\left(\mathbf{r}^{\prime \prime}\right)}{\left|\mathbf{r}-\mathbf{r}^{\prime \prime}\right|} d V^{\prime \prime}
$$

where $\rho^{\prime}=\lambda \sum_{i} e_{i} c_{i}{ }^{\prime}$. Since $c_{i}{ }^{\prime}$ depends on $\lambda$ and $T$ only through the variable $\lambda \psi^{\prime} / T$, according to the Boltzmann expression, $c_{i}{ }^{\prime}=n_{i} \exp \left(-\lambda e_{i} \psi^{\prime} / k T\right) /$ $\int \exp \left(-\lambda e_{\imath} \psi^{\prime} / k T\right) d V$, it follows that $\rho^{\prime}$ equals $\lambda$ times a function of $\lambda \psi^{\prime} / T$. Let $\rho^{\prime}=\lambda f\left(\lambda \psi^{\prime} / T\right)$. Throughout, regardless of the value of $\lambda$ and $T$, the polymer configuration and its charge distribution are held constant in integrating these expressions for $F_{e}{ }^{\prime}$; that is, $\rho_{p}{ }^{\prime} / \lambda$ $=\alpha_{r} C_{p}$ is independent of $\lambda$ and of $T$. Thus it follows from

$$
\frac{\lambda \psi^{\prime}}{T}=-\frac{1}{D} \int \frac{\lambda^{2}}{T}\left[\frac{f\left(\lambda \psi^{\prime} / T\right)+\rho_{p}^{\prime} / \lambda}{\left|\mathbf{r}-\mathbf{r}^{\prime \prime}\right|}\right] d V
$$

that $\lambda \psi^{\prime} / T$ depends only on $\lambda^{2} / T$ at constant $D$.

Since $E_{e}{ }^{\prime}=\frac{1}{2} \int \psi^{\prime}\left(\rho^{\prime}+\rho_{p}{ }^{\prime}\right) d V$ we conclude from

$$
\frac{E_{e}{ }^{\prime}}{T}=\frac{1}{2} \int \frac{\lambda \psi^{\prime}}{T}\left[f\left(\lambda \psi^{\prime} / T\right)+\rho_{p}^{\prime} / \lambda\right] d V
$$

that $E_{e}{ }^{\prime} / T$ is a function only of $\lambda \psi^{\prime} / T$ and therefore, at constant $D$, only a function of $\lambda^{2} / T$. Since

$$
F_{e}^{\prime} / T=\int_{\lambda=0}^{\lambda=\lambda} \frac{2 E_{e}^{\prime}}{T} \frac{d \lambda}{\lambda},
$$

$F_{e}^{\prime} / T$ is, at constant $D$, only a function of $\lambda^{2} / T$, since $d \lambda / \lambda$ can for integration purposes be expressed in terms of the derivative of a function (logarithm) of $\lambda^{2} / T$. This establishes the statement that the assumption of the Poisson-Boltzmann equation does not affect the equality of Eqs. (6) and (8).

\section{(ii) Comparison of Eqs. (6) and (11)}

It has been established in the previous section that the use of the Poisson-Boltzmann Eq. (1) does not affect the identity

$$
E_{e}=\left(\frac{\partial F_{e} / T}{\partial 1 / T}\right)_{D}=F_{e}-\left(\frac{\partial F_{e}}{\partial T}\right)_{D}
$$

where $F_{e}$ is given by Eq. (6). Using this result it may be concluded that it is sufficient to show that $\left[\partial F_{e} / \partial T\right]_{D}$ $=-\left(S-S_{i d}\right)$ in order to demonstrate that the use of Eq. (1) does not render Eqs. (6) and (11) inconsistent. The entropy difference $\left(S-S_{i d}\right)$ is computed from Eqs. (9) and (10). In the following proof all differentia- tions with respect to $T$ will be performed at constant $\lambda$ and $D$, and those with respect to $\lambda$, at constant $T$ and $D$.

We have from the differentiation of (6)

$\frac{\partial F_{e}}{\partial T}=\iint\left[\left(\rho^{\prime}+\rho_{p}{ }^{\prime}\right) \frac{\partial \psi^{\prime}}{\partial T}+\psi^{\prime}\left(\frac{\partial \rho^{\prime}}{\partial T}+\frac{\partial \rho_{p}{ }^{\prime}}{\partial T}\right)\right] \frac{d \lambda}{\lambda} d V$.

Expressing $\psi^{\prime}$ and $\partial \psi^{\prime} / \partial T$ in terms of $\rho^{\prime}$ and $\partial \rho^{\prime} / \partial T$ with the aid of $\left(12^{\prime}\right)$, it may be verified that Eq. (47) becomes

$$
\frac{\partial F_{e}}{\partial T}=2 \iint \psi^{\prime}\left(\frac{\partial \rho^{\prime}}{\partial T}+\frac{\partial \rho_{p}{ }^{\prime}}{\partial T}\right) \frac{d \lambda}{\lambda} d V .
$$

Since the polymer configuration is kept constant in integrating the expression for $F_{e}, \partial \rho_{p}^{\prime} / \partial T=0$ and

$$
\frac{\partial F_{e}}{\partial T}=2 \iint \psi^{\prime} \frac{\partial \rho^{\prime}}{\partial T} \frac{d \lambda}{\lambda} d V .
$$

Since $\rho^{\prime}$ is $\lambda$ times a function of $\lambda^{2} / T$, it may be verified that

$$
\frac{1}{\lambda} \frac{\partial \rho^{\prime}}{\partial T}=\frac{\partial}{\partial T}\left(\rho^{\prime} / \lambda\right)=-\frac{\lambda}{2 T} \frac{\partial}{\partial \lambda}\left(\rho^{\prime} / \lambda\right)
$$

by expressing each partial derivative in terms of a derivative with respect to $\lambda^{2} / T$. Substituting this into Eq. (49) and integrating by parts, we have

$$
\frac{\partial F_{e}}{\partial T}=-\frac{\int \rho \psi d V}{T}+\iint\left(\frac{\rho^{\prime}}{T} \frac{\partial \psi^{\prime}}{\partial \lambda}+\frac{\rho^{\prime} \psi^{\prime}}{\lambda T}\right) d \lambda d V .
$$

This expression may be simplified by employing the following identity [established by carrying out the differentiation on the left-hand side of $(52)$ and then introducing the Boltzmann expression for $\left.c_{i}{ }^{\prime}\right]$ :

$$
k \frac{\partial}{\partial \lambda} \sum_{i} n_{i} \ln V_{i}^{\prime}=-\int\left(\frac{\rho^{\prime}}{T} \frac{\partial \psi^{\prime}}{\partial \lambda}+\frac{\rho^{\prime} \psi^{\prime}}{\lambda T}\right) d V .
$$

Integrating this from $\lambda=0$ to $\lambda=1$ the left-hand side becomes $k \sum_{i} n_{i} \ln \left(V_{i} / V\right)$ since $V_{i}{ }^{\prime}=V_{i}$ when $\lambda=1$ and $V_{i}^{\prime}=V$ when $\lambda=0$. Thus from Eqs. (51) and (52) it follows that

$$
\frac{\partial F_{e}}{\partial T}=-\frac{\int \rho \psi d V}{T}-k \sum_{i} n_{i} \ln \frac{V_{i}}{V} .
$$

Since according to (2)

$$
-\frac{\rho \psi}{k T}=-\frac{\sum_{i} e_{i} c_{i} \psi}{k T}=\sum_{i} c_{i} \ln \frac{c_{i} V_{i}}{n_{i}}
$$


it follows that from (53)

$$
\begin{aligned}
\frac{\partial F_{e}}{\partial T} & =k \sum_{i} \int c_{i} \ln c_{i} d V-k \sum_{i} n_{i} \ln \frac{n_{i}}{V} \\
& =k \sum_{i} \int\left(c_{i} \ln c_{i}-c_{i}\right) d V-k \sum_{i}\left(n_{i} \ln \frac{n_{i}}{V}-n_{i}\right), \\
& =-\left(S-S_{i d}\right) .
\end{aligned}
$$

\section{APPENDIX II}

\section{Calculation of Distribution Functions and of Effect of Redistribution of Groups on $\delta F$}

It will be shown here that the redistribution of the charges on the polymer and, incidentally, the change in the configurational variable $h$, both of which accompany a reversible change in state, contribute nothing to the calculated free-energy change $\delta F$, so that $\delta F$ equals that estimated at fixed relative distribution, $\alpha_{r} / \alpha$ and at fixed $h$.

On the basis of Eq. (3) for $F$ and the various equations given in the text for the terms which occur there, we may write

$$
F=\int K d V-T S_{p}
$$

where

$$
\begin{aligned}
K=k T C_{p}\left[\alpha_{r} \ln \alpha_{r}+\left(1-\alpha_{r}\right) \ln \left(1-\alpha_{r}\right)\right] \\
+\frac{1}{2} \psi\left(\sum_{i} c_{i} e_{i}+\alpha_{r} C_{p} e_{p}\right)+k T \sum_{i}\left(c_{i} \ln c_{i}-c_{i}\right) \\
+\sum_{i} c_{i} \mu_{i}{ }^{0}+C_{p}\left[\alpha_{r} \mu_{A}{ }^{0}+\left(1-\alpha_{r}\right) \mu_{H A}{ }^{0}\right]
\end{aligned}
$$

It follows from $\alpha_{r} C_{p}=\left(\alpha_{r} / \alpha\right) C_{p} \alpha$ and $c_{i}=\left(c_{i} / n_{i}\right) n_{i}$ that $\delta\left(\alpha_{r} C_{p}\right)={ }_{\alpha c} C_{p} \delta\left(\alpha_{r} / \alpha\right)+\left(\alpha_{r} / \alpha\right) C_{p} \delta \alpha+\alpha_{r} \delta C_{p}$ and $\delta c_{i}$ $=n_{i} \delta\left(c_{i} / n_{i}\right)+\left(c_{i} / n_{i}\right) \delta n_{i}$. That is, the change in $c_{i}$ for example may be written as the sum of a change in $n_{i}$ at fixed relative distribution $c_{i} / n_{i}$, plus a change in the relative distribution. We may then write for $\delta F$,

$$
\begin{gathered}
\delta F=\int\left[\frac{\partial K}{\partial\left(\alpha_{r} / \alpha\right)} \delta\left(\frac{\alpha_{r}}{\alpha}\right)\right. \\
\left.+\sum_{i} \frac{\partial K}{\partial\left(c_{i} / n_{i}\right)} \delta\left(\frac{c_{i}}{n_{i}}\right)+\frac{\partial K}{\partial C_{p}} \frac{d C_{p}}{d h} \delta h\right] d V \\
+\delta \int(K)_{\alpha_{r} / \alpha, c_{i} / n_{i}, C_{p}} d V-T \frac{d S_{p}}{d h}
\end{gathered}
$$

where in the partial derivative $\partial K / \partial\left(\alpha_{r} / \alpha\right)$, for example, $\alpha, c_{i} / n_{i}, n_{i}, C_{p}$ and all thermodynamic variables are held fixed. The variation in the second integral is performed at fixed $\alpha_{r} / \alpha, c_{i} / n_{i}$, and $C_{p}$, as indicated by the subscripts, and only differs from zero when there is a change in $\alpha$ or in the thermodynamic state. Actually, because of the equilibrium relation $\partial F / \partial \alpha=0$, it follows therefore, that in a given thermodynamic state it equals zero. At equilibrium in a specified thermodynamic state $\delta F$ equals zero and the variations in $\alpha_{r} / \alpha, c_{i} / n_{i}$ and $C_{p}$ are subject to the restrictions of constant number of each type of ion, and constant number of neutralized and of unneutralized groups on the polymer. Therefore, in a given state

$$
\left.\begin{array}{c}
\frac{1}{\alpha} \int \delta\left(\alpha_{r} C_{p}\right) d V=0 \\
\frac{1}{n_{i}} \int \delta c_{i} d V=0 \text { for all } i \\
\int \delta C_{p} d V=0
\end{array}\right\} .
$$

Multiplying these equations by the constants $A, B_{i}$, and $C$, the Lagrangian multipliers, and adding to (58), where the second integral and $\delta F$ are set equal to zero, it follows that the coefficients of $\delta\left(\alpha_{r} / \alpha\right), \delta\left(c_{i} / n_{i}\right)$ and $\delta h$ are zero since this new equation is obeyed for arbitrary variations in $\alpha_{r} / \alpha, c_{i} / n_{i}$, and $h$. [At least these variations in $\alpha_{r} / \alpha$ and $c_{i} / n_{i}$ are arbitrary subject to (59) and this limitation may be removed by imagining $V$ spanned by cells, the integral of $K$ over $V$ becoming a sum over cells, and choosing $A$, and $B_{i}$ so that the coefficient of the variations in a particular cell is zero. The variations in $\alpha_{r} / \alpha$ and $c_{i} / n_{i}$ in the other cells are arbitrary so that the coefficients of these variations must equal zero in these cells too if the equation is to be obeyed for all variations.] By this process Eqs. (60), (61), and (62) defining the equilibrium $\alpha_{r} / \alpha, c_{i} / n_{i}$, and $h$ are obtained

$$
\begin{array}{r}
\frac{\partial K}{\partial\left(\alpha_{r} / \alpha\right)}+C_{p} A=0 \\
\frac{\partial K}{\partial\left(c_{i} / n_{i}\right)}+B_{i}=0 \\
\int\left(\frac{\partial K}{\partial C_{p}}+A \frac{\alpha_{r}}{\alpha}+C\right) \frac{d C_{p}}{d h} d V-T \frac{d S_{p}}{d h}=0 .
\end{array}
$$

Introducing these results for $\partial K / \partial\left(\alpha_{r} / \alpha\right)$ and $\partial K / \partial C_{p}$ etc., into (58) it follows that

$$
\begin{aligned}
\delta F=-A \int \delta\left(\frac{\alpha_{r} C_{p}}{\alpha}\right) d V-C \int & \delta C_{p} d V \\
& +\delta \int(K)_{\alpha_{r} / \alpha_{1}, C_{p}} d V
\end{aligned}
$$

where we have combined the term involving $\partial K / \partial\left(c_{i} / n_{i}\right)$ with the last term in Eq. (58). In Eq. (63) the first term is really $-A$ multiplied by $m \delta(\alpha / \alpha)$, which always 
equals zero ${ }^{19}$ while the second term is also always zero. Therefore,

$$
\delta F=\delta \int(K)_{\alpha_{r} / \alpha, C_{p}} d V=\delta(F)_{\alpha_{r} / \alpha, C_{p}}
$$

which establishes the desired result.

Introducing expression (57) for $K$ into (60), (61), and (62) it follows that $\alpha$ is given by Boltzmann expression (2), that $\alpha_{r}$ is given by (65), where $D$ is a constant

${ }^{19}$ Actually,

$0=m \delta(\alpha / \alpha)=\delta \int\left(C_{p} \alpha_{s}\right) / \alpha d V=\alpha_{r}{ }^{3} C_{p}{ }^{s} \delta V / \alpha+\int \delta\left(\alpha_{r} C_{p} / \alpha\right) d V$

and $C_{p}{ }^{8}$ equals zero since the polymer lies within $V$ and not on the bounding surface, $S$, of this volume, so that this expression for $m \delta(\alpha / \alpha)$ becomes $\int \delta\left(\alpha_{r} C_{p} / \alpha\right) d V$. estimated by multiplying

$$
\alpha_{r}=\frac{D e^{-e_{p} \psi / k T}}{1+D e^{-e_{p} \psi / k T}}
$$

both sides of $(65)$ by $C_{p} d V$ and integrating over $V$,

$$
m \alpha=\int C_{p} \alpha_{r} d V=D \int \frac{e^{-e_{p} \psi / k T}}{1+D e^{-e_{p} \psi / k T}} d V
$$

and that $h$ is the solution of

$$
\int\left(k T \ln \alpha_{r}+e_{p} \psi\right) \frac{d C_{p}}{d h} d V=T-\frac{d S_{p}}{d h} .
$$

\title{
Photochemical Studies of the Porphyrins. III. Photoreduction of a Porphyrin by Benzoin
}

\author{
Gilbert R. Seely* and Melvin Calvin \\ Department of Chemistry and Radiation Laboratory, $\dagger$ University of California, Berkeley, California
}

(Received October 18, 1954)

\begin{abstract}
An examination of the kinetics of the photoreduction of zinc tetraphenylporphin by benzoin is reported. The products are successively the dihydro- (chlorin) derivative; a tetrahydro-derivative and possibly a hexahydro compound as well. It is demonstrated that the primary requirement is the photoactivation of the benzoin; a secondary reaction, involving photoactivated porphyrin as well, is also demonstrated. The quantum yield based on light absorbed by benzoin varies between 0.01 and 0.06 .
\end{abstract}

W ORK has been previously reported from this laboratory ${ }^{1-3}$ on the photochemical oxidation of zinc dihydrotetraphenylporphin, also known as zinc tetraphenylchlorin. Due to interest in the role of chlorophyll in photosynthesis, and the possibility that reduction or oxidation of chlorophyll may have a function in that role, we have continued study of photochemical reactions of simple porphyrins.

Since zinc tetraphenylchlorin had been quantitatively oxidized to the porphin by quinone, ${ }^{1}$ when irradiated by light absorbed by the chlorin, we sought to reverse this reaction by photochemically reducing zinc tetraphenylporphin. It was found that, using the following reducing agents in deoxygenated benzene,

\footnotetext{
* This paper was abstracted from the thesis of Gilbert R. Seely, submitted in partial fulfillment of the requirement for Doctor of Philosophy, University of California, Berkeley, February, 1954. Present address: 9771 TSU, Det. 3, Dugway Proving Grounds, Utah.

$\dagger$ The work described in this paper was supported by the U. S. Atomic Energy Commission.

${ }^{1}$ G. D. Dorough, Ph.D. thesis, University of California, Berkeley, 1947; M. Calvin and G. D. Dorough, J. Am. Chem. Soc. 70, 699 (1948); G. D. Dorough and M. Calvin, Science 105, 433 (1947).

2 Ball, Calvin, and Dorough, J. Am. Chem. Soc. 68, 2278 (1946).

${ }^{3} \mathrm{~F}$. M. Huennekens, $\mathrm{Ph} . \mathrm{D}$. thesis, University of California, Berkeley, 1948; F. M. Huennekens and M. Calvin, J. Am. Chem.
} Soc. 71, 4024, 4031 (1949). alcohol, or pyridine solutions, reduction could be detected spectroscopically after exposure to sunlight: benzoin, $p$-dimethylaminobenzoin, ascorbic acid, dihydroxyacetone, dioxymaleic acid, reductone, hydrazobenzene, semicarbazide, $n$-hexyl mercaptan, 1,2-ethanedithiol and arabinose. The rapidity and extent of reduction varied greatly with the reducing agent used; with benzoin, reduction was spectroscopically complete after 15 or 20 minutes of sunlight. The mercaptans and arabinose produced only a trace of zinc tetraphenylchlorin after days of exposure to sunlight. The hydrazines worked very little faster, and the hydroxyketones listed (except benzoin), required several hours, or even days for significant reduction.

In general, reduction was found to occur in two steps. At first, a band near $625 \mathrm{~m} \mu$, indicative of zinc tetraphenylchlorin, was seen to emerge. Later, a band near $600 \mathrm{~m} \mu$ intensified, which is indicative of a compound which will be identified as a zinc tetraphenyltetrahydroporphin. On prolonged exposure to light, this band, too, fades, leaving a washed-out spectrum.

The reduction by benzoin was the only one fast enough to permit kinetic measurements under artificial illumination, so this reagent was used throughout, for determination of the quantum yield and the mechanism of the reduction. 